\title{
TERAPIA PRIMARIA EN APLASIA DE MÉDULA ÓSEA \\ Trasplante alogénico de progenitores hematopoyéticos vs. terapia inmunosupresora
}

Kenny Mauricio Gálvez MD*, María Helena Solano MD**, Carlos Alberto Ramírez MD***, Virginia Abello MD****, Enrique Pedraza MD*****, Hermann Esguerra MD******, Carmen Rosales MD*******, Licet Villamizar $* * * * * * * *$, Jesús Antonio Reyes $* * * * * * * * *$

\section{Resumen}

Antecedentes: la aplasia de médula ósea (AMO) es una enfermedad poco frecuente caracterizada por un síndrome de falla medular con citopenias periféricas y médula ósea hipo o acelular, en ausencia de blastos y mielodisplasia. El trasplante alogénico de progenitores hematopoyéticos (TAPH) y la terapia inmunosupresora (TI) son tratamientos aceptados para pacientes con esta patología. Objetivo: determinar la supervivencia global a un año de los pacientes con AMO de acuerdo con el tratamiento recibido. Diseño: estudio de serie de casos. Lugar: servicio de hematología del Hospital de San José y unidad de trasplante de médula ósea de la Clínica de Marly. Pacientes: 36 con AMO. Intervención: trasplante alogénico de progenitores hematopoyéticos de un donante HLA-idéntico relacionado o terapia inmunosupresora. Medición: se realizó el análisis descriptivo a través de tablas de frecuencia. Las probabilidades de supervivencia fueron estimadas usando el método de Kaplan-Meier. Resultados: la supervivencia global a un año del grupo de TAPH fue de $64 \%$ y del grupo de TI $57 \%$. Respecto a los factores pronósticos, se encontró que en los dos grupos de tratamiento los recuentos de neutrófilos $>\mathbf{5 0 0}$ y de plaquetas $>\mathbf{1 0 . 0 0 0}$ al momento del diagnóstico se relacionaron con una mayor supervivencia en el primer año. Además un menor soporte transfusional tanto de $P Q$ como de glóbulos rojos desde el diagnóstico hasta el momento del tratamiento tiene una fuerte relación con la supervivencia al año. Conclusión: en los pacientes con AMO el TAPH y la TI conllevan tasas de supervivencia a un año muy similares.

Palabras clave: aplasia médula ósea, trasplante alogénico, terapia inmunosupresora, supervivencia, factores pronósticos.

Abreviaturas: AMO, aplasia de médula ósea; PQ, plaquetas; TAPH, trasplante alogénico de progenitores hematopoyécticos; TI, terapia inmunosupresora; HLA, antígeno mayor de histocompatibilidad.

\footnotetext{
Fecha recibido: marzo 20 de 2007 - Fecha aceptado: junio 5 de 2007

* Residente de hematología, Fundación Universitaria de Ciencias de la Salud.

** Jefe del servicio de hematología, Hospital de San José. Profesor titularFundación Universitaria de Ciencias de la Salud.

*** Profesor de hematología Fundación Universitaria de Ciencias de la Salud. Jefe de hemodinamia en Colsánitas a nivel nacional.

***** Hematóloga Fundación Universitaria de Ciencias de la Salud. Unidad de trasplante de médula ósea Clínica de Marly.
}

\footnotetext{
*****Hematólogo Fundación Universitaria de Ciencias de la Salud. Unidad de Trasplante de médula ósea Clínica de Marly.

*******Oncólogo unidad de trasplante de médula ósea, Clínica de Mary.

********Hematóloga unidad de trasplante de médula ósea Cínica de Marly

*********Epidemióloga división de investigaciones Fundación Universitaria de Ciencias de la Salud.

**********Bioestadístico división de investigaciones Fundación Universitaria de Ciencias de la Salud.
} 


\section{Introducción}

La AMO es una enfermedad poco frecuente caracterizada por un síndrome de falla medular con citopenias periféricas y médula ósea hipo o acelular, en ausencia de blastos y mielodisplasia. ${ }^{1}$ La AMO severa se define según los criterios del grupo internacional de estudio de la AMO, ${ }^{1,2}$ como la presencia de por lo menos dos de los tres siguientes criterios: 1) recuento de neutrófilos menor de $500 \mathrm{cel} \times 10^{9} / \mathrm{L}$, 2) recuento de reticulocitos corregidos menor del $1 \%$ y 3 ) recuento de PQ menor de $20 \times 10^{9} / \mathrm{L}$. Los pacientes con criterios para AMO severa pero con recuento de neutrófilos menor de 200 cel x $10^{9} / \mathrm{L}$ son clasificados en el grupo de aplasia de AMO muy severa y si el recuento de neutrófilos es mayor de 500 cel x 109/L se define como AMO no severa. Hasta hace cerca de 30 años los tratamientos utilizados en el manejo de esta entidad tenían una tasa de éxito entre el 10 a $35 \%$ y la mayoría de pacientes morían como consecuencia de las complicaciones propias de la enfermedad, tales como sangrado e infecciones severas. ${ }^{3}$ En los últimos años el tratamiento y la supervivencia de los pacientes con AMO ha cambiado con la introducción del TAPH y con el perfeccionamiento de la $\mathrm{TI}^{4}{ }^{4}$ Con respecto al trasplante, los factores que permitieron este avance han sido la utilización de condicionamiento con base en ciclofosfamida/globulina antitimocito, la profilaxis de la enfermedad injerto contra huésped con ciclosporina más metotrexate y el uso de productos sanguíneos irradiados y filtrados. En los pacientes menores de 50 años con aplasia severa que tienen donantes con HLA idéntico, el TAPH generalmente se considera el tratamiento de elección. En aquellos mayores de 50 años la morbilidad y mortalidad relacionada con el trasplante contraindica el procedimiento. Para ellos, al igual que para quienes no tienen donante HLA idéntico, la TI es la terapia de primera línea. Existen varios trabajos que confirman la utilidad de la TI, como el del grupo europeo e italiano de médula ósea, en donde se siguieron 100 pacientes con AMO tratados con linfoglobulina, ciclosporina, prednisona y filgrastim encontrando respuestas hematológicas del $77 \% .{ }^{5}$ Otro estudio que incluyó tanto niños como adultos mostró una respuesta del $62 \%$ a los 43 meses y una supervivencia a cinco años de $86 \% .{ }^{6}$ Con respecto al TAPH una de las más grandes series publicadas es la del registro internacional de trasplante de médula ósea, en el cual se incluyeron 1699 pacientes que recibieron trasplante de hermanos HLA compatibles entre 1991 y 1997, donde las probabilidades de supervivencia a cinco años por edad fueron: menores de 20 años, $75 \%$, entre los 21 y 39 años, 68\%; y mayores de 40 años, 65\%. ${ }^{7}$

En este estudio se muestra la experiencia de dos centros de referencia de Bogotá en el tratamiento de la AMO con TAPH o TI, se resumen los resultados de cada tratamiento, se examinan los factores pronósticos asociados a supervivencia y las complicaciones relacionadas con cada una de las terapias. El objetivo es determinar la supervivencia global a un año de los enfermos con aplasia de médula ósea de acuerdo con el tratamiento recibido.

\section{Métodos}

Selección de pacientes: se revisaron las historias clínicas de todos los pacientes con diagnóstico confirmado de aplasia medular por mielograma y biopsia de médula ósea, tratados en los servicios de hematología del Hospital de San José y de trasplante de médula ósea de la Clínica de Marly entre el $1^{\circ}$ de enero de 1993 y el 31 de agosto de 2005. Se realizaron diferentes exámenes de laboratorio con el fin de descartar una etiología secundaria de la aplasia, como pruebas para hepatitis viral B y C, VIH, anticuerpos antinucleares, anti DNA de doble cadena, anti Ro, anti $\mathrm{La}$, anti Sm, niveles séricos de vitamina B12, ácido fólico y TSH, prueba de HAM, prueba de sucrosa y cariotipo estresado con mitomicina C. Se excluyeron los pacientes que no tuvieron seguimiento mayor de un año, cuyo diagnóstico no fue confirmado según los criterios internacionales y aquellos diagnosticados en las instituciones participantes del estudio pero que no fueron tratados en ellas.

Diseño: estudio de serie de casos. 


\section{Regímenes de tratamiento}

Grupo de TAPH: se utilizaron varios protocolos de condicionamiento como ciclofosfamida, ciclofosfamida más linfoglobulina y fludarabina-ciclofosfamida-campath. Los regímenes profilácticos para la enfermedad injerto contra huésped (EICH) fueron: ciclosporina, ciclosporina más metotrexate y ciclosporina-metotrexate-prednisona. ${ }^{8,9}$ La severidad de la EICH aguda fue determinada según los criterios previamente descritos por Glucksberg y Sullivan. ${ }^{10,11}$

Grupo de TI: los pacientes recibieron GAT (linfoglobulina $20 \mathrm{mg} / \mathrm{kg} /$ día o timoglobulina $15 \mathrm{mg} / \mathrm{kg} /$ día por 5 días) más ciclosporina ( $5 \mathrm{mg} / \mathrm{kg} /$ día). Se administró prednisona a todos los pacientes con el fin de prevenir o suprimir las manifestaciones clínicas de la enfermedad del suero.

Los pacientes de ambos grupos que cumplían los criterios de neutropenia febril recibieron tratamiento empírico con antibióticos de amplio espectro. Se utilizó soporte transfusional con hemoderivados leucorreducidos si el nivel de hemoglobina era menor de $8 \mathrm{~g} / \mathrm{dl}$ y/o si el recuento de plaquetas era menor de 10.000 o el paciente presentaba sangrado.

Análisis estadístico: se realizó el análisis descriptivo a través de tablas de frecuencia. Las probabilidades de supervivencia fueron estimadas usando el método de Kaplan-Meier. ${ }^{12}$ Todos los datos de supervivencia fueron censurados con la última fecha de contacto.

\section{Resultados}

Se revisaron 45 historias clínicas. En total 26 pacientes recibieron trasplante alogénico. Cuatro fueron excluidos debido a que no completaron el año de seguimiento. De 19 pacientes con terapia inmunosupresora, se excluyeron cinco por no completar el año de seguimiento. En la Tabla 1

\begin{tabular}{|l|c|}
\hline \multicolumn{2}{|c|}{ Tabla I. Característica del grupo con AMO. } \\
\hline \multicolumn{1}{|c|}{ Característica } & Pacientes con AMO n=36 \\
\hline $\begin{array}{l}\text { Causa de la aplasia } \\
\text {-Idiopática n (\%) } \\
\text {-Anemia de Fanconi n (\%) }\end{array}$ & $27(68 \%)$ \\
-HPN* n (\%) & I (6\%) \\
-Tóxicos n (\%) & $1(6 \%)$ \\
\hline Severidad de la aplasia & \\
-Muy severa n (\%) & $7(13.6 \%)$ \\
-Severa n (\%) & $19(59 \%)$ \\
-No severa $n$ (\%) & $10(27.4 \%)$ \\
\hline Mediana neutrófilos al diagnóstico (rango) & $35(20-900)$ \\
\hline Mediana PQ al diagnóstico (rango) & $12.000(3.000-39.000)$ \\
\hline Mediana transfusiones de glóbulos rojos antes del tratamiento (rango) & $8(0-19)$ \\
\hline Mediana transfusiones de PQ antes del tratamiento (rango) & $4(0-20)$ \\
\hline
\end{tabular}

* HPN: hemoglobinuria paroxística nocturna. 
se presentan las características de la totalidad de pacientes con aplasia medular.

Grupo de trasplante alogénico de progenitores hematopoyéticos: el promedio de edad fue de 19 años (rango de 4 a 57), la mitad era de sexo masculino. La relación sexo donante/receptor fue la siguiente: masculino/masculino 4 (18\%), masculino/femenino 9 (41\%), femenino/masculino 7 (32\%) y femenino/femenino dos. Nueve $(41 \%)$ pacientes recibieron otros tratamientos previos al trasplante; 3 (13.6\%) GAT, 3 (13.6\%) corticoides, $2(9 \%)$ corticoides más filgrastim y $1(4.5 \%)$ eritropoyetina más filgrastim. Se utilizaron varios protocolos de condicionamiento pretrasplante, $11(50 \%)$ pacientes recibieron ciclofosfamida más GAT, 7 (32\%) ciclofosfamida y 4 (18\%) fludarabina, ciclofosfamida más alemtuzumab. La profilaxis de la enfermedad injerto contra huésped se realizó con ciclosporina en $2(9 \%)$ pacientes, ciclosporina más metotrexate en $12(68 \%)$ y con ciclosporina, metotrexate y prednisona en 5 (23\%). El injerto de neutrófilos se logró en promedio 18 días después de la infusión (rango de 8 a 54 días). Dos (9\%) pacientes perdieron el injerto. Las principales complicaciones tempranas (antes de 100 días) fueron infecciosas: seis (27.4\%) con bacteriemia por gram positivos, dos (9\%) neumonía, dos $(9 \%)$ choque séptico, dos (9\%) infección micótica, dos $(9 \%)$ sepsis por gram negativos y uno (4.5\%) presentó sepsis por catéter; hemorrágicas: tres pacientes, uno hemorragia alveolar, uno sangrado intracerebral y uno hemorragia vítrea; 16 (73\%) presentaron EICHa; siete (32\%) grado I, cuatro (18\%) grado II, uno (4.5\%)

\begin{tabular}{|c|c|c|}
\hline Característica & $\begin{array}{l}\text { TAPH* } \\
\mathrm{n}=22\end{array}$ & $\begin{array}{l}\mathrm{T} \mid * * \\
\mathrm{n}=14\end{array}$ \\
\hline $\begin{array}{l}\text { Causa de la aplasia } \\
\text {-Idiopática n (\%) } \\
\text {-Anemia de Fanconi n (\%) } \\
\text {-HPN*** n (\%) } \\
\text {-Tóxicos n (\%) }\end{array}$ & $\begin{array}{c}15(68 \%) \\
7 \text { (32\%) } \\
--- \\
---\end{array}$ & $\begin{array}{c}12(88 \%) \\
--- \\
\text { I (6\%) } \\
\text { I }(6 \%)\end{array}$ \\
\hline $\begin{array}{l}\text { Severidad de la aplasia } \\
\text {-Muy severa } n(\%) \\
\text {-Severa } n(\%) \\
\text {-No severa } n(\%)\end{array}$ & $\begin{array}{l}3(13.6 \%) \\
13(59 \%) \\
6(27.4 \%)\end{array}$ & $\begin{array}{l}4(28.6 \%) \\
6(42.8 \%) \\
4(28.6 \%)\end{array}$ \\
\hline Mediana,neutrófilos al diagnóstico (rango) & $340(100-900)$ & $385(20-825)$ \\
\hline Mediana, PQ al diagnóstico (rango) & $\begin{array}{l}12.000(3.000- \\
28.000)\end{array}$ & $\begin{array}{l}14.000(3.000 \\
39.000)\end{array}$ \\
\hline $\begin{array}{l}\text { Mediana,transfusiones de glóbulos rojos antes del tratamiento } \\
\text { (rango) }\end{array}$ & $7(0-19)$ & $8(2-14)$ \\
\hline Mediana, transfusiones de PQ antes del tratamiento (rango) & $3(0-20)$ & $6(0-18)$ \\
\hline
\end{tabular}

* TAPH: trasplante alogénico de progenitores hematopoyéticos.

** TI: terapia inmunosupresora.

*** HPN: hemoglobinuria paroxística nocturna. 
grado III y cuatro (18\%) grado IV. Otras características se ilustran en la Tabla 2.

Grupo de terapia inmunosupresora: el promedio de edad fue de 44 años (rango de 18 a 60). Ocho $(57 \%)$ de sexo masculino y seis $(43 \%)$ femenino. Con respecto al tratamiento utilizado, doce (86\%) recibieron linfoglobulina y dos (14\%) timoglobulina. El promedio de aplasia después del inicio del tratamiento fue de 64.5 días (rango de 32 a 124 días). Tres $(21 \%)$ pacientes desarrollaron enfermedad del suero asociada con la infusión de GAT. Diez (71\%) toxicidad secundaria al uso de ciclosporina, dada por hipertensión arterial; cuatro (29\%) aumento de los azoados secundario al uso del medicamento. Otras características son ilustradas en la Tabla 2.

\section{Supervivencia}

La curva de supervivencia de acuerdo con cada grupo de tratamiento se ilustra en la Figura 1. La global a un año del grupo de TAPH fue de $64 \%$ y del grupo de TI $57 \%(p=0.39)$. Las causas de muerte en el grupo de TAPH fueron infección $13.6 \%$ (tres pacientes), EICHa 9\% (dos), sangrado $4.5 \%$ (uno) y pérdida del injerto $9 \%$ (dos). Con respecto al grupo de TI la causa de muerte fue sangrado en cuatro pacientes $(28 \%)$ e infección en dos (14\%). En la Tabla 3 se muestra la causa y tiempo de muerte con relación al grupo de TAPH.

\section{Factores pronósticos y supervivencia}

La relación entre factores pronósticos y supervivencia global al año son ilustradas en la Tabla 4.

\section{Discusión}

En este estudio de serie de casos se encontró que en los pacientes con $\mathrm{AMO}$ que recibieron tratamiento con TAPH tienen una supervivencia a un año de $64 \%$ y los de TI 57\%, sin una diferencia estadística significativa, similar a lo reportado en otros estudios. ${ }^{5,6,7}$ Con respecto a este resultado es importante tener en cuenta que en el grupo de TI no se logró completar

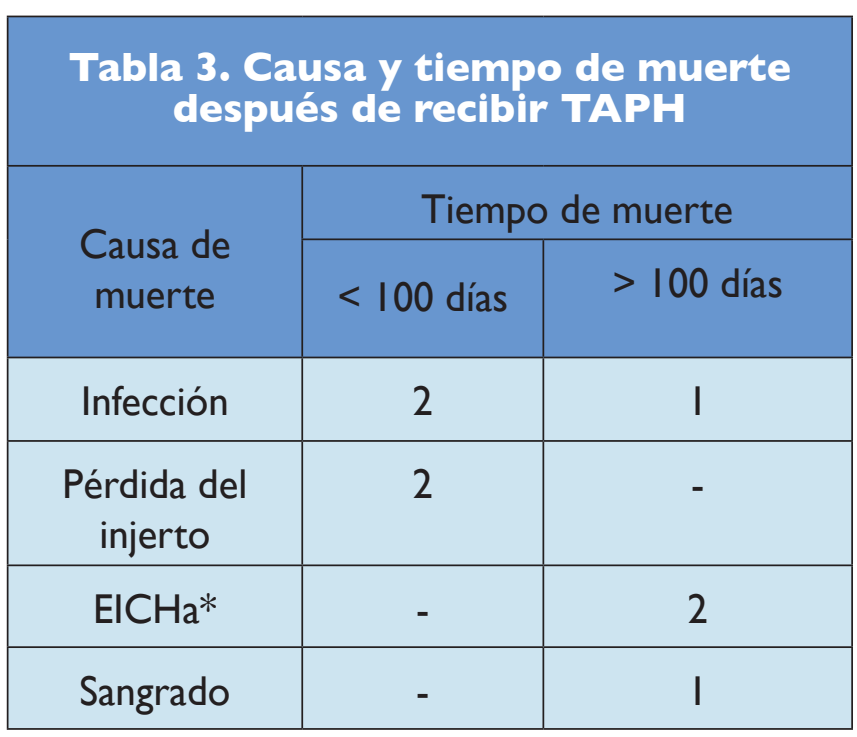

* EICHa: enfermedad injerto contra huésped aguda.

el número de pacientes que se calculó al principio en el tamaño de la muestra, lo cual no permite llegar a una conclusión con respecto a esta diferencia.

En cuanto a los factores pronósticos, en concordancia con otros estudios, ${ }^{13,14}$ se encontró que en los dos grupos de tratamiento los recuentos de neutrófilos $>500$ y de $\mathrm{PQ}>10.000$ al diagnóstico se relacionaron con una mayor supervivencia en el primer año. De la misma manera un menor soporte transfusional tanto de PQ como de glóbulos rojos desde el diagnóstico hasta el momento del tratamiento, tiene una fuerte relación con la supervivencia al año.

Estos hallazgos son consistentes con un análisis de 509 pacientes con AMO reportados por el European Bone Marrow Transplant Registry. ${ }^{15} 218$ recibieron trasplante alogénico de donante compatible y $291 \mathrm{TI}$ con GAT. La supervivencia a cinco años no difirió entre los dos grupos. El recuento bajo de neutrófilos se asoció con disminución de la supervivencia. El soporte transfusional previo al tratamiento no se analiza en esta publicación.

En un estudio realizado en adultos ( $>16$ años), investigadores de la Universidad de California compararon 56 pacientes con AMO que recibieron GAT con 55 sometidos a TAPH. ${ }^{16}$ La supervivencia a seis años no difirió entre los dos grupos, $49 \%$ para GAT y $52 \%$ para 


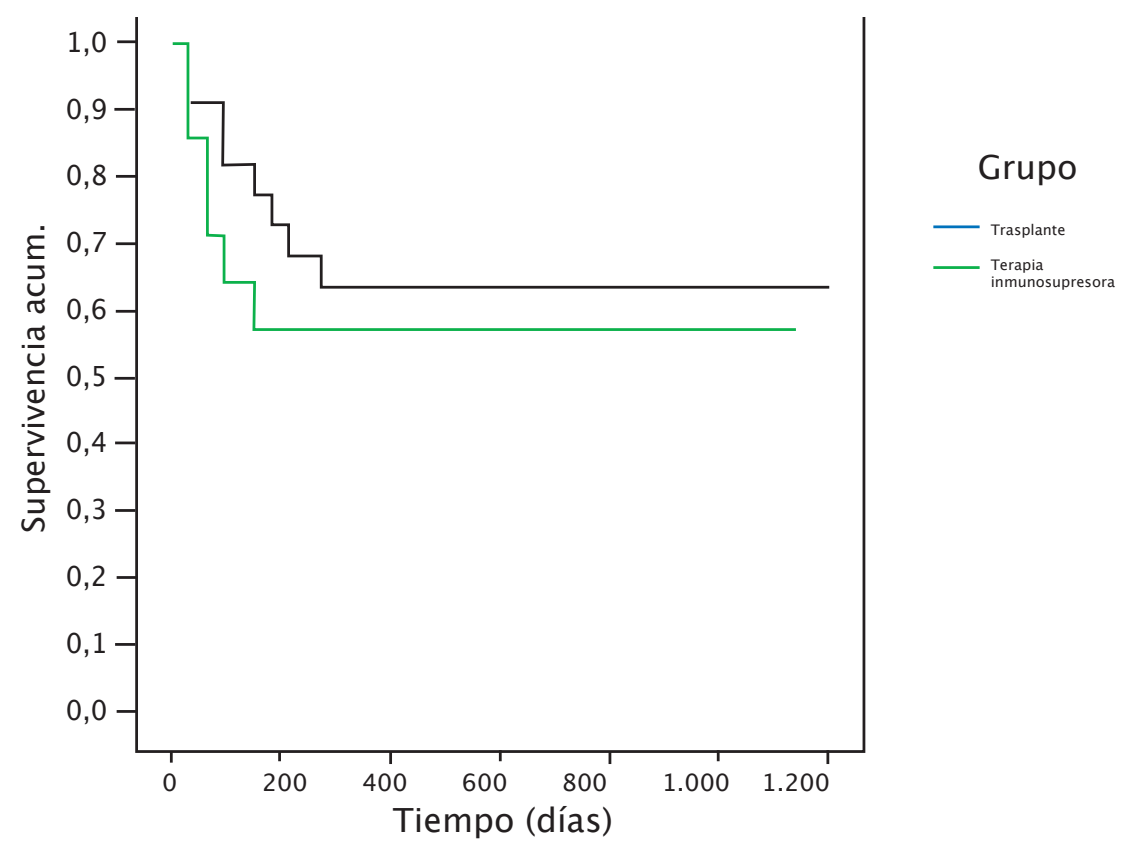

Figura 1. Curva de supervivencia de acuerdo a cada grupo de tratamiento por el método de Kaplan-Meir $(p=0.39)$.

trasplante. En este último la supervivencia fue mejor en los pacientes tratados después del año 1983, lo que coincide con profilaxis más efectiva de la EICH.

International Bone Marrow Transplant Registry (IBMTR) reportó que los pacientes con AMOS que habían recibido más de 20 transfusiones antes del trasplante, tenían una supervivencia menor comparados con los que habían recibido menos de 20 transfusiones o no habían sido transfundidos. ${ }^{17}$

Uno de los factores pronósticos mencionados con frecuencia en estudios de AMO es la edad. ${ }^{18}$ En nuestro estudio no pareció ser un factor relacionado con la supervivencia, sin embargo es probable que se deba al tamaño pequeño de la muestra. La mortalidad en el grupo de trasplante se relacionó en esencia con infección y enfermedad injerto contra huésped. La falla en el injerto, que es muy frecuente en los politransfundidos, ${ }^{19}$ se presentó en dos pacientes (9\%), porcentaje similar a lo reportado en otros centros (5 al 15\%).

El principal factor que determina la decisión de tratar un paciente con AMO con TAPH o TI es la dispo- nibilidad de un donante relacionado HLA idéntico. ${ }^{20}$ Aunque se describen remisiones espontáneas tardías, es de vital importancia iniciar inmediatamente después del diagnóstico un tratamiento encaminado a restaurar la hematopoyesis normal y disminuir al máximo la necesidad de soporte transfusional, debido a que aumenta el riesgo de rechazo al injerto y disminuye la supervivencia cualquiera que sea el tratamiento. En el caso de pacientes con donante se debe restringir el uso de hemoderivados de parientes cercanos, para evitar el riesgo de aloinmunización y por lo tanto pérdida del injerto. ${ }^{21,22}$

El desarrollo de nuevos métodos de laboratorio y terapéuticos y el descubrimiento de similitudes entre la AMO y otras enfermedades mediadas por células $\mathrm{T}$, han llevado a comprender mejor los mecanismos capaces de producir fallo de la función medular y avanzar en el conocimiento y tratamiento de esta patología. Los progresos futuros dependen de la realización de estudios multicéntricos prospectivos, por lo que los pacientes con AMO deben ser referidos a hospitales especializados con experiencia en esta enfermedad. 


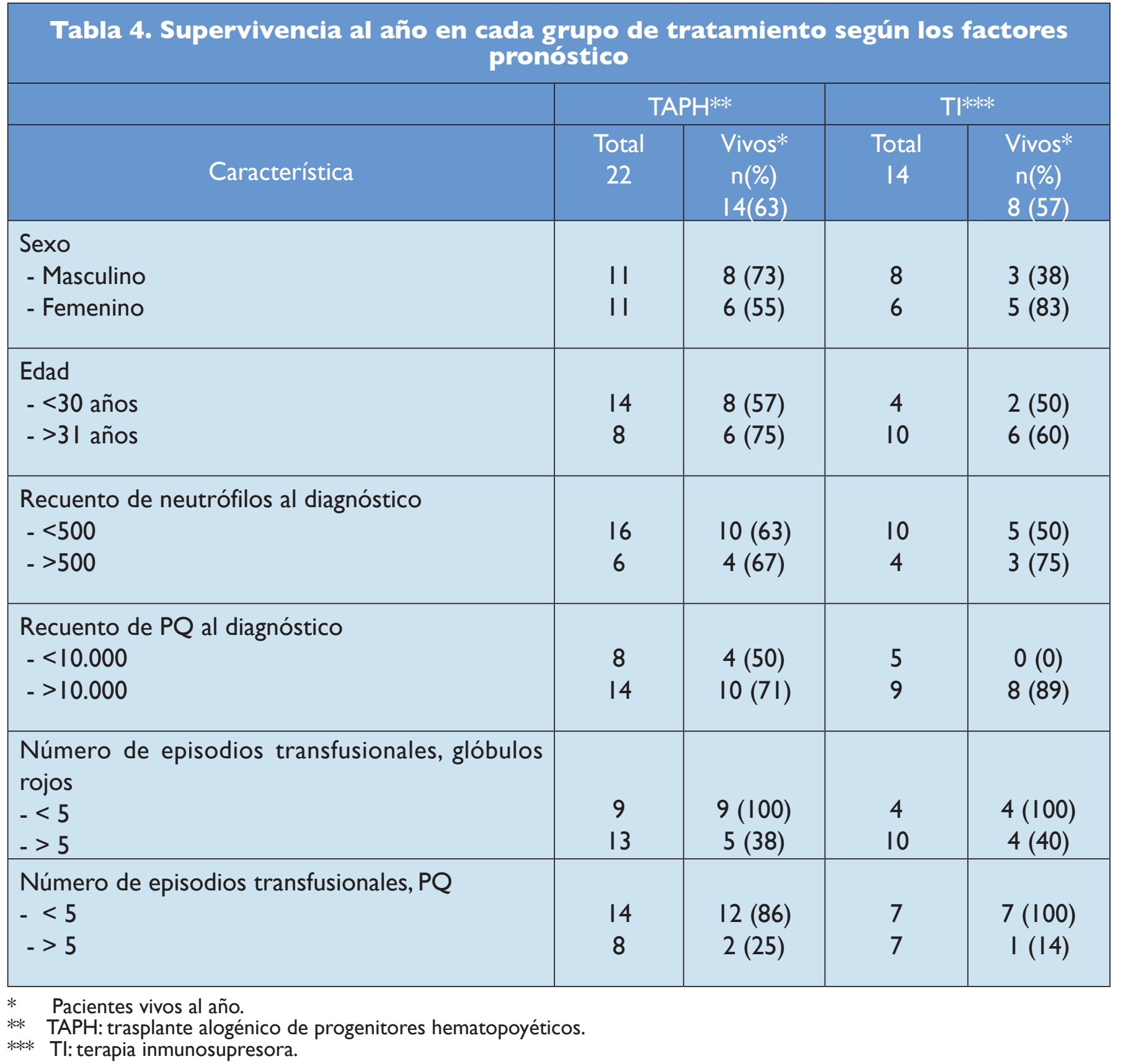

El presente estudio es una serie de casos y sólo pretende describir la experiencia de dos grupos en el manejo de pacientes con AMO. Este tipo de publicación es muy útil para formular hipótesis, pero no sirve para evaluar o probar la presencia de una asociación estadística y se limita a la simple identificación y descripción de un conjunto de casos clínicos en un intervalo de tiempo. Sólo estudios prospectivos con mayor tiempo de seguimiento, permitirán llegar a conclusiones definitivas sobre los factores de riesgo y el mejor método de tratamiento para esta patología poco común. Sin embargo teniendo en cuenta la baja prevalencia del padecimiento es poco probable que este tipo de estudio se pueda realizar, a no ser en cooperación con muchas otras instituciones en el país o la región.

\section{Referencias}

1- British Committee for Standards in Haematology (BCSH) General Haematology Task Force. Guidelines for the diagnosis and management of acquired aplastic anaemia. Br J Haematol 2003;123:782-801.

2- Camitta BM, Thomas ED, Nathan DG, Santos G, Gordon-Smith EC, Gale RP, Rappeport JM, Storb R. Severe 
aplastic anemia: a prospective study of the effect of early marrow transplantation on acute mortality. Blood. 1976 Jul;48(1):63-70.

3- Young NS. Aplastic anaemia. Lancet. 1995 Jul 22;346(8969):228-32.

4- Bacigalupo A, Brand R, Oneto R, Bruno B, Socie G, Passweg J, Locasciulli A, Van Lint MT, Tichelli A, McCann S, Marsh J, Ljungman P, Hows J, Marin P, Schrezenmeier H. Treatment of acquired severe aplastic anemia: bone marrow transplantation compared with immunosuppressive therapy--The European Group for Blood and Marrow Transplantation experience.Semin Hematol. 2000 Jan;37(1):69-80.

5- Bacigalupo A, Bruno B, Saracco P, Di Bona E, Locasciulli A, Locatelli F, Gabbas A, Dufour C, Arcese W, Testi G, Broccia G, Carotenuto M, Coser P, Barbui T, Leoni P, Ferster A. Antilymphocyte globulin, cyclosporine, prednisolone, and granulocyte colony-stimulating factor for severe aplastic anemia: an update of the GITMO/EBMT study on 100 patients. European Group for Blood and Marrow Transplantation (EBMT) Working Party on Severe Aplastic Anemia and the Gruppo Italiano Trapianti di Midolio Osseo (GITMO). Blood. 2000 Mar 15;95(6):1931-4.

6- Frickhofen N, Rosenfeld SJ. Immunosuppressive treatment of aplastic anemia with antithymocyte globulin and cyclosporine. Semin Hematol. 2000 Jan;37(1):56-68.

7- Horowitz MM. Current status of allogenic bone marrow transplantation in acquired aplastic anemia. Semin hematol 2000; 37:30-42.

8- Storb R, Deeg HJ, Farewell V, Doney K, Appelbaum F, Beatty P, Bensinger W, Buckner CD, Clift R, Hansen J, et al. Marrow transplantation for severe aplastic anemia: methotrexate alone compared with a combination of methotrexate and cyclosporine for prevention of acute graft-versus-host disease. Blood. 1986 Jul;68(1):119-25.

9- Storb R, Leisenring W, Deeg HJ, Anasetti C, Appelbaum F, Bensinger W, Buckner CD, Clift R, Doney K, Hansen J, et al. Long-term follow-up of a randomized trial of graft-versus-host disease prevention by methotrexate/cyclosporine versus methotrexate alone in patients given marrow grafts for severe aplastic anemia. Blood. 1994 May 1;83(9):274950.

10- Glucksberg H, Storb R, Fefer A, Buckner CD, Neiman PE, Clift RA, et al. Clinical manifestations of graft-versus-host disease in human recipients of marrow from HLA-matched sibling donors. Transplantation. 1974; 18:295-304.

11- Sullivan KM, Agura E, Anasetti C, Appelbaum F, Badger C, Bearman S, Erickson K, Flowers M, Hansen J, Loughran T, et al. Chronic graft-versus-host disease and other late complications of bone marrow transplantation. Semin Hematol. 1991 Jul;28(3):250-9.

12- Kaplan EL, Meler P. Nonparametric estimation from incomplete observations. J Am Stat Assoc 1958;53:45781.

13- Gluckman E, Rokicka-Milewska R, Hann I, Nikiforakis E, Tavakoli F, Cohen-Scali S, Bacigalupo A; European Group for Blood and Marrow Transplantation Working Party for Severe Aplastic Anemia. Results and follow-up of a phase III randomized study of recombinant human-granulocyte stimulating factor as support for immunosuppressive therapy in patients with severe aplastic anaemia. Br J Haematol. 2002 Dec;119(4):1075-82.

14- Viollier R, Passweg J, Gregor M, Favre G, Kuhne T, Nissen C, Gratwohl A, Tichelli A. Quality-adjusted survival analysis shows differences in outcome after immunosuppression or bone marrow transplantation in aplastic anemia.Ann Hematol. 2005 Jan;84(1):47-55.

15- Bacigalupo A, Hows J, Gluckman E, Nissen C, Marsh J, Van Lint MT, Congiu M, De Planque MM, Ernst P, McCann S, et al. Bone marrow transplantation (BMT) versus immunosuppression for the treatment of severe aplastic anaemia (SAA): a report of the EBMT SAA working party. Br J Haematol. 1988 Oct;70(2):177-82.

16- Paquette RL, Tebyani N, Frane M, Ireland P, Ho WG, Champlin RE, Nimer SD. Long-term outcome of aplastic anemia in adults treated with antithymocyte globulin: comparison with bone marrow transplantation. Blood. 1995 Jan 1;85(1):283-90.

17- Schrezenmeier H, Bredeson C, Bruno B, Loberiza FR, Camitta B, Oneto R, et al. Comparison of allogeneic bone marrow and peripheral blood stem cell transplantation for aplastic anaemia: collaborative study of European Blood and Marrow Transplant Group (EBMT) and International Bone Marrow Transplant Registry (IBMTR). Blood 2003267.

18- Gluckman E, Horowitz MM, Champlin RE, Hows JM, Bacigalupo A, Biggs JC, Camitta BM, Gale RP, GordonSmith EC, Marmont AM, et al. Bone marrow transplantation for severe aplastic anemia: influence of conditioning and graft-versus-host disease prophylaxis regimens on outcome. Blood. 1992 Jan 1;79(1):269-75.

19- Passweg JR, Pérez WS, Eapen M, Camitta BM, Gluckman E, Hinterberger W, Hows JM, Marsh JC, Pasquini R, Schrezenmeier H, Socié G, Zhang MJ, Bredeson C. Bone marrow transplants from mismatched related and unrelated donors for severe aplastic anemia. Bone Marrow Transplant. 2006 Apr;37(7):641-9.

20- Kojima S, Matsuyama T, Kato S, Kigasawa H, Kobayashi R, Kikuta A, Sakamaki H, Ikuta K, Tsuchida M, Hoshi Y, Morishima Y, Kodera Y. Outcome of 154 patients with severe aplastic anemia who received transplants from unrelated donors: the Japan Marrow Donor Program. Blood. 2002 Aug 1;100(3):799-803.

21- Mao P, Zhu Z, Wang H, Wang S, Mo W, Ying Y, Li Q, Xu Y. Sustained and stable hematopoietic donor-recipient mixed chimerism after unrelated cord blood transplantation for adult patients with severe aplastic anemia. Eur J Haematol. 2005 Nov;75(5):430-5.

22- Ades L, Mary JY, Robin M, Ferry C, Porcher R, Esperou H, Ribaud P, Devergie A, Traineau R, Gluckman E, Socié $\mathrm{G}$. Long-term outcome after bone marrow transplantation for severe aplastic anemia. Blood. 2004 Apr 1;103(7):24907. 